\title{
Características biológicas de Trichospilus diatraeae (Hymenoptera: Eulophidae) nos hospedeiros Bombyx mori (Lepidoptera: Bombycidae) e Diatraea saccharalis (Lepidoptera: Crambidae)
}

\author{
Vanessa Rodrigues Ferreira Calado ${ }^{{ }^{*}}$ \\ Fabricio Fagundes Pereira ${ }^{2}$ \\ Elizangela Leite Vargas ${ }^{1}$ \\ Daniele Fabiana Glaeser $^{3}$ \\ Fabiana Garcia de Oliveira ${ }^{2}$ \\ Universidade Federal da Grande Dourados \\ ${ }^{1}$ Faculdade de Ciências Agrárias \\ Caixa Postal 533, CEP 79840-970, Dourados, MS $\square$ Brasil \\ ${ }^{2}$ Faculdade de Ciências Biológicas e Ambiental \\ ${ }^{3}$ Empresa Brasileira de Pesquisa Agropecuária, Embrapa Agropecuária Oeste \\ * Autor para correspondência \\ vanrodriguescalado@gmail.com
}

Submetido em 20/03/2013

Aceito para publicação em 19/12/2013

\section{Resumo}

Trichospilus diatraeae Cherian \& Margabandhu, 1942 (Hymenoptera: Eulophidae) é um endoparasitoide pupal de lepidópteros e tem sido estudado como agente potencial no controle biológico de pragas. Para o desenvolvimento de técnicas de criação de parasitoides, é necessária a escolha do hospedeiro alternativo adequado, assim, o objetivo deste artigo é avaliar as características biológicas de T. diatraeae em relação aos hospedeiros Bombyx mori Linneaus (Lepidoptera: Bombycidae) e Diatraea saccharalis (Fabricius) (Lepidoptera: Crambidae), em laboratório. Doze pupas de B. mori e doze pupas D. saccharalis, com 72 e $24 \mathrm{~h}$ de vida, respectivamente, foram expostas ao parasitismo por 21 fêmeas parasitoides a $25 \pm 1^{\circ} \mathrm{C}$, com umidade relativa de $70 \pm 10 \%$ e fotofase de $14 \mathrm{~h}$. A duração do ciclo de vida (ovo $\square$ adulto) de $T$. diatraeae foi de 19,44 $\pm 0,12$ dias em pupas de D. saccharalis e 18,00 $\pm 0,05$ dias em pupas de B. mori, a emergência de progênie do parasitoide foi $66,60 \%$ em pupas de $D$. saccharalis e 75,00\% em pupas de B. mori. A progênie de T. diatraeae foi de 354,50 \pm 43,21 por pupa de $D$. saccharalis e $469,11 \pm 15,19$ por pupa de $B$. mori. Trichospilus diatraeae demonstrou adequabilidade ao hospedeiro e sua capacidade de parasitar diferentes hospedeiros.

Palavras-chave: Controle biológico; Desempenho reprodutivo; Endoparasitoide

\section{Abstract}

Biological characteristics of Trichospilus diatraeae (Hymenopetra: Eulophidae) in the hosts Bombyx mori (Lepidoptera: Bombycidae) and Diatraea saccharalis (Lepidoptera: Crambidae). Trichospilus diatraeae 
Cherian \& Margabandhu, 1942 (Hymenoptera: Eulophidae) is a pupal endoparasitoid of lepidoptera and it has been studied as a potential agent for biological control of pests. For developing techniques to breed parasitoids, there is a need to choose the appropriate alternative host, thus, this article aims to evaluate the biological characteristics of $T$. diatraeae with regard to the hosts Bombyx mori Linneaus (Lepidoptera: Bombycidae) and Diatraea saccharalis (Fabricius) (Lepidoptera: Crambidae), in laboratory. Twelve pupae of B. mori and twelve pupae of $D$. saccharalis, within 72 and $24 \mathrm{~h}$ of life, respectively, were exposed to parasitism by 21 female parasitoids at $25 \pm 1{ }^{\circ} \mathrm{C}$, with relative humidity $70 \pm 10 \%$ and photophase of $14 \mathrm{~h}$. Life-cycle duration (egg $\square$ adult) of $T$. diatraeae was $19.44 \pm 0.12$ days in pupae of $D$. saccharalis and $18.00 \pm 0.05$ days in pupae of B. mori, the emergence of parasitoid progeny was $66.60 \%$ in pupae of $D$. saccharalis, and $75.00 \%$ in pupae of $B$. mori. The progeny of $T$. diatraeae was $354.50 \pm 43.21$ per pupa of $D$. saccharalis and $469.11 \pm 15.19$ per pupa of B. mori. Trichospilus diatraeae showed suitability to the host and its ability to parasitize various hosts.

Key words: Biological control; Endoparasitoid; Reproductive performance

\section{Introdução}

O endoparasitoide de pupas de lepidópteros Trichospilus diatraeae Cherian \& Margabandhu, 1942 (Hymenoptera: Eulophidae) tem sido relatado e estudado como agente potencial no controle biológico de pragas agrícolas e florestais de várias espécies de lepidópteros das famílias Erebidae, Noctuidade, Geometridae e Sartuniidae (PARON; BERTI FILHO, 2000; OLIVEIRA et al., 2001; PEREIRA et al., 2008; ZACHÉ et al., 2010a; 2010b; 2011a; 2011b; 2012; MELO et al., 2011; PASTORI et al., 2012).

A dificuldade de criação contínua de alguns hospedeiros e presas naturais de parasitoides e de predadores em laboratório exige que esses inimigos naturais sejam criados em hospedeiros e presas alternativos ou de substituição, os quais normalmente não são preferidos no campo, embora promovam suficiente desenvolvimento ao mesmo (PARRA et al., 2002; ZANUNCIO et al., 2008; PEREIRA et al., 2009; 2010a). O desenvolvimento de técnicas de criação de parasitoides para programas de controle biológico depende da escolha do hospedeiro alternativo adequado (PRATISSOLI et al., 2005), que poderá reduzir custos de produção e aumentar a eficiência de programas de criação massal (DIAS et al., 2008; PEREIRA et al., 2010b).

A capacidade reprodutiva de $T$. diatraeae foi avaliada em pupas de Diatraea saccharalis (Fabricius, 1794) (Lepidoptera: Crambidae), Anticarsia gemmatalis Hübner, 1818 (Lepidoptera: Erebidae), Spodoptera frugiperda (Smith, 1797), Heliothis virescens Fabricius,
1781 (Lepidoptera: Noctuidae) (PARON; BERTI FILHO, 2000; ANDRADE et al., 2011); Thyrinteina arnobia (Stoll, 1782) (Lepidoptera: Geometridae), Hylesia paulex Hübner, 1820 (Lepidoptera: Sartuniidae) (PASTORI et al., 2012) e Spodoptera cosmioides Walker, 1858 (Lepidoptera: Noctuidae) (ZACHÉ et al., 2012).

Pupas de Bombyx mori Linneaus, 1758 (Lepidoptera: Bombycidae) apresentam alto valor proteico (WANGDUN et al., 2004), podem ser criadas em grandes quantidades e, apesar de serem encerradas em casulos, podem ser facilmente retiradas e manipuladas (PEREIRA et al., 2009; 2010b) e já foram indicadas como hospedeiro alternativo efeciente de Palmistichus elaeisis Delvare \& La Salle, 1993 (Hymenoptera: Eulophidae) (PEREIRA et al., 2009), motivos pelos quais se resolveu utilizar pupas dessa espécie como hospedeiro alternativo de T. diatraeae e comparar o seu desenvolvimento com o hospedeiro natural $D$. saccharalis.

O objetivo deste trabalho foi avaliar o desenvolvimento e as características biológicas de $T$. diatraeae nos hospedeiros B. mori e D. saccharalis.

\section{Material e Métodos}

\section{Local de condução dos experimentos}

Os experimentos foram realizados no Laboratório de Entomologia/Controle Biológico (LECOBIOL), Faculdade de Ciências Agrárias (FCA) da Universidade Federal da Grande Dourados (UFGD), em Dourados, Mato Grosso do Sul. 


\section{Criação de Diatraea saccharalis (Lepidoptera: Crambidae)}

Foram utilizados insetos oriundos da criação do Laboratório de Entomologia/Controle Biológico da Faculdade de Ciências Agrárias da UFGD com a seguinte metodologia: após a eclosão, as lagartas desse lepidóptero foram colocadas em frascos de vidro $(10 \mathrm{x}$ $22 \mathrm{~cm}$ ) com tampa de plástico teladas, com dieta artificial segundo PARRA (2007), com adaptações (Tabela 1), onde permaneceram até a formação de pupas. As pupas foram recolhidas, selecionadas para bioensaios e o restante transferido para gaiolas de PVC $(10 \times 22 \mathrm{~cm})[50$ adultos (20 machos e 30 fêmeas)] revestido com folhas de papel sulfite umedecido, servindo como substrato para oviposição. As gaiolas de PVC foram fechadas com tecido do tipo $\square$ voil $\square$ e elástico, conforme proposto por PARRA (2007).

TABELA 1: Componentes utilizados para dieta artificial de Diatraea saccharalis (Lepidoptera: Crambidae), segundo PARRA (2007), com adaptações.

\begin{tabular}{cc}
\hline Componentes & \\
\hline Farelo de soja & $300 \mathrm{~g}$ \\
Germe de trigo & $150 \mathrm{~g}$ \\
Levedura de Cana & $225 \mathrm{~g}$ \\
Açúcar & $205 \mathrm{~g}$ \\
Nipagin & $15 \mathrm{~g}$ \\
Ácido Ascórbico & $8 \mathrm{~g}$ \\
Sais de Wesson & $15 \mathrm{~g}$ \\
Cloreto de colina & $2 \mathrm{~g}$ \\
Caragenato & $65 \mathrm{~g}$ \\
Vita Gold & $3 \mathrm{~mL}$ \\
Solução Vitamínica & $40 \mathrm{~mL}$ \\
Formol & $6 \mathrm{~mL}$ \\
Tetraciclina & $2 \mathrm{~g}$ \\
Ácido Nalidíxico & $2 \mathrm{~g}$ \\
\hline
\end{tabular}

${ }^{1}$ niacinamida: $1,0 \mathrm{~g}$; pantotenato de cálcio: $1,0 \mathrm{~g}$; riboflavina: 0,50 $\mathrm{g}$; tiamina: $0,5 \mathrm{~g}$; piridoxina: $0,25 \mathrm{~g}$; ácido fólico: $0,10 \mathrm{~g}$; biotina: 0,02 mg; vitamina B12 (1000 mg/mL):2,00 mL.

\section{Criação de Bombyx mori}

Ovos de B. mori foram fornecidos pela Empresa Bratac $\AA$. Lagartas recém-eclodidas foram colocadas em bandejas plásticas $(39,3 \times 59,5 \times 7,0 \mathrm{~cm})$ contendo folhas de amoreira (Morus alba L.) as quais foram trocadas diariamente até a formação dos casulos. Posteriormente esses foram transferidos para bandejas plásticas $(28,3 \mathrm{x}$ $36,0 \times 7,0 \mathrm{~cm}$ ) e acondicionados em câmara climatizada tipo B.O.D a $25 \pm 1^{\circ} \mathrm{C}, 70 \pm 10 \%$ de umidade relativa e fotofase de $12 \mathrm{~h}$ (PEREIRA et al., 2009).

\section{Criação de Trichospilus diatraeae}

Adultos de T. diatraeae oriundos da criação do Laboratório de Entomologia/Controle Biológico da Faculdade de Ciências Agrárias da UFGD foram mantidos em tubos de vidro $(8,5 \times 2,5 \mathrm{~cm})$ fechados com algodão e alimentados com gotículas de mel puro. Para manutenção da criação, pupas de $D$. saccharalis com $24 \mathrm{a} 48 \mathrm{~h}$ foram expostas ao parasitismo por $72 \mathrm{~h}$. Após esse período pupas parasitadas foram individualizadas e mantidas a $25 \pm 1{ }^{\circ} \mathrm{C}$, umidade relativa de $70 \pm 10 \% \mathrm{e}$ fotofase de $14 \mathrm{~h}$ até a emergência de adultos.

\section{Bioensaios}

Doze pupas de $D$. saccharalis com peso médio de $160 \pm 0,01 \mathrm{mg}$ e com idade de 24 horas e doze pupas $B$. mori com peso médio de $800 \pm 0,01 \mathrm{mg}$, e com $72 \mathrm{~h}$ de idade, foram individualizadas em tubos de vidro ( $14 \mathrm{x}$ $2,2 \mathrm{~cm}$ ) e expostas ao parasitismo por 21 fêmeas de $T$. diatraeae com $48 \mathrm{~h}$ de idade. $\mathrm{O}$ fato de utilizar-se 21 fêmeas parasitoides por pupa de cada hospedeiro, foi devido ao tamanho e peso superior da pupa de $B$. mori em relação à pupa de $D$. saccharalis.

As fêmeas do parasitoide foram retiradas dos tubos após $24 \mathrm{~h}$ e as pupas parasitadas mantidas em câmara climatizada tipo B.O.D. a $25 \pm 1^{\circ} \mathrm{C}$ umidade relativa de $70 \pm 10 \%$ e fotofase de $14 \mathrm{~h}$ até a emergência da progênie.

O delineamento experimental foi o inteiramente casualizado com dois tratamentos representados por $T$. diatraeae obtidos a partir dos hospedeiros $D$. saccharalis ou $B$. mori com 12 repetições, sendo cada uma representada por uma pupa hospedeira.

As características biológicas avaliadas foram: número de pupas parasitadas (pupas parasitadas por tratamento, descontando-se a mortalidade natural do hospedeiro) (ABBOTT, 1925); número de pupas 
com emergência da progênie; número de parasitoides emergidos por pupa de $D$. saccharalis e B. mori (progênie), duração do ciclo de vida (ovo-adulto) do parasitoide; longevidade de adultos (para avaliação dessa variável foram selecionados ao acaso 20 fêmeas e 10 machos de $T$. diatraeae recém emergidos por tratamento, os quais foram individualizados em tubos de ensaio contendo mel até a sua morte); a largura da cápsula cefálica (foram escolhidos ao acaso em cada tratamento, 15 fêmeas e 15 machos de $T$. diatraeae, objetivando medir a largura da cápsula cefálica em ocular micrométrica); e razão sexual ( $\mathrm{RS}=$ número de fêmeas/ número de adultos) de $T$. diatraeae. A sexagem dos parasitoides foi determinada de acordo com características morfológicas da antena e do abdome.

Os valores do número de pupas parasitadas ou com emergência de $T$. diatraeae foram submetidos à análise de modelos lineares generalizados com distribuição binomial $(\mathrm{P} \leq 0,05)$ com o R Statistical System (IHAKA; GENTLEMAN, 1996). Essa análise foi realizada com os dados originais não-paramétricos, representados por 0 (pupas não parasitadas e pupas sem emergência) e 1 (pupas parasitadas e pupas com emergência). Os dados da duração do ciclo de vida, do número de parasitoides emergidos por pupa de D. saccharalis e de B. mori (progênie), da razão sexual, da longevidade e da largura da cápsula cefálica de fêmeas e machos de $T$. diatraeae foram submetidos à análise de variância.

\section{Resultados}

O parasitismo de $T$. diatraeae em pupas de $B$. mori e $D$. saccharalis foi de $100 \%(\mathrm{P}<0,01)$, com emergência de $75 \%$ e $66,6 \%(\mathrm{P}<0,01)$ para o primeiro e segundo hospedeiro, respectivamente (Figura 1). A possibilidade de criação de $T$. diatraeae em pupa de $B$. mori é relatada pela primeira vez neste trabalho.

A duração do ciclo de vida $(\mathrm{F}=0,025 \mathrm{P}=0,8761)$, a razão sexual $(\mathrm{F}=0,360 ; \mathrm{P}=0,5591)$, o tamanho da cápsula cefálica das fêmeas $(\mathrm{F}=4,507 ; \mathrm{P}=0,0434)$ e dos machos $(\mathrm{F}=1,084 ; \mathrm{P}=0,3115)$ e o tamanho do corpo de fêmeas $(\mathrm{F}=2,862 ; \mathrm{P}=0,1018)$ e machos $(\mathrm{F}=3,963$; $\mathrm{P}=0,0619)$ de $T$. diatraeae em pupas de $B$. mori e $D$. saccharalis foram semelhantes (Tabela 2). Entretanto, a progênie $(\mathrm{F}=18,383 ; \mathrm{P}=0,0009)$ de $T$. diatraeae foi maior em pupas de $B$. mori (Tabela 2 ) e a longevidade de fêmeas $(\mathrm{F}=44,986 ; \mathrm{P}=0,0000)$ e de machos $(\mathrm{F}=36,422$; $\mathrm{P}=0,0000)$ do parasitoide proveniente de pupas de B. mori diferiu daquelas apresentada por $T$. diatraeae emergidos de pupas de D. saccharalis (Tabela 2).

FIGURA 1: Porcentagem de parasitismo e de emergência de Trichospilus diatraeae (Hymenopetra: Eulophidae) em pupas de Diatraea saccharalis (Lepidoptera: Crambidae) e de Bombyx mori (Lepidoptera: Bombycidae) a temperatura de $25 \pm 2{ }^{\circ} \mathrm{C}, 70 \pm 10 \%$ de umidade relativa e fotofase de $14 \mathrm{~h}$. Parasitismo $(\mathrm{P}<0,01)$ Emergência $(\mathrm{P}<0,01)$.

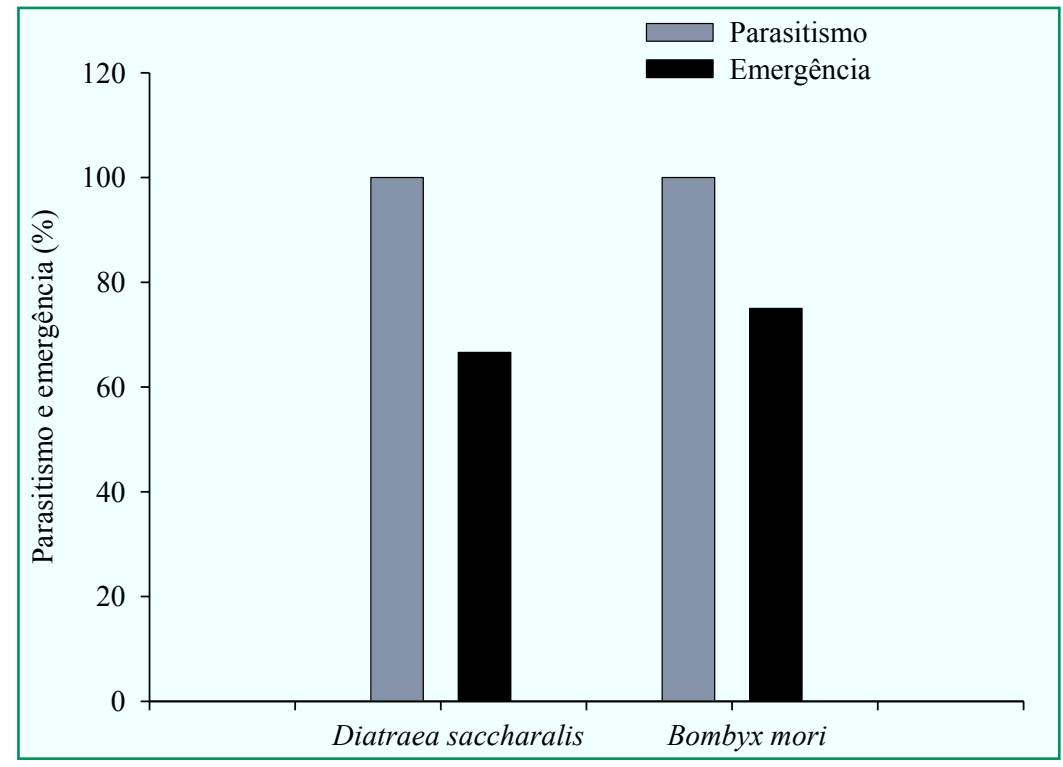


TABELA 2: Características biológicas (Médias \pm EP) ${ }^{1}$ de Trichospilus diatraeae (Hymenopetra: Eulophidae) em pupas de Bombyx mori (Lepidoptera: Bombycidae) e de Diatraea saccharalis (Lepidoptera: Crambidae), em laboratório à temperatura de $25 \pm 2{ }^{\circ} \mathrm{C}, 70 \pm 10 \%$ de umidade relativa e fotofase de $14 \mathrm{~h}$ (n= Número de parasitoides).

\begin{tabular}{lcccc}
\hline \multicolumn{1}{c}{ Características biológicas } & Bombyx mori & n & Diatraea saccharalis & n \\
\hline Duração do ciclo de vida (dias) & $19,44 \pm 0,09 \mathrm{a}$ & 09 & $18,00 \pm 0,05 \mathrm{a}$ & 08 \\
Progênie por pupa & $469,11 \pm 15,19 \mathrm{a}$ & 09 & $354,50 \pm 41,88 \mathrm{~b}$ & 08 \\
Cápsula cefálica do macho (mm) & $0,30 \pm 0,03 \mathrm{a}$ & 10 & $0,31 \pm 0,04 \mathrm{a}$ & 10 \\
Cápsula cefálica da fêmea (mm) & $0,35 \pm 0,03 \mathrm{~b}$ & 20 & $0,38 \pm 0,03 \mathrm{a}$ & 20 \\
Tamanho do corpo da fêmea (mm) & $1,09 \pm 0,09 \mathrm{a}$ & 20 & $1,06 \pm 0,08 \mathrm{a}$ & 20 \\
Tamanho do corpo do macho (mm) & $0,978 \pm 0,05 \mathrm{a}$ & 10 & $0,971 \pm 0,05 \mathrm{a}$ & 10 \\
Longevidade da Fêmea (dias) & $25,80 \pm 3,15 \mathrm{a}$ & 20 & $17,70 \pm 3,23 \mathrm{~b}$ & 20 \\
Longevidade do Macho (dias) & $22,40 \pm 2,97 \mathrm{a}$ & 10 & $13,00 \pm 2,97 \mathrm{~b}$ & 10 \\
Razão sexual & $0,85 \pm 0,07 \mathrm{a}$ & 09 & $0,84 \pm 0,03 \mathrm{a}$ & 08 \\
\hline
\end{tabular}

${ }^{1}$ Médias seguidas de mesma letra minúscula na mesma linha não diferem entre si, pela análise de variância.

\section{Discussão}

O parasitismo de pupas de B. mori e D. saccharalis por $T$. diatraeae demonstra a capacidade do parasitoide em aceitar e se adequar a hospedeiros diferentes dos seus preferenciais na natureza. Resultados semelhantes foram relatados para $T$. diatraeae em pupas de $A$. gemmatalis, S. frugiperda, H. virescens (PARON; BERTI FILHO, 2000), T. arnobia, H. paulex (PASTORI et al., 2012) e $S$. cosmioides (ZACHÉ et al., 2012). O índice de emergência da progênie de $T$. diatraeae em pupas de B. mori foi maior do que em pupas de D. saccharalis e isso pode está relacionado com a capacidade de suporte e disponibilidade de recurso alimentar do hospedeiro para o parasitoide, uma vez que pupas de B. mori são maiores que aquelas de $D$. saccharalis ou quando o recurso é finito e não renovável, como no caso de ovos, lagartas e pupas, a fêmea deposita ovos férteis de acordo com a quantidade de recurso (PANNIZI; PARRA, 1991). Tais características podem também justificar a maior progênie de $T$. diatraeae em pupas de $B$. mori do que em pupas de $D$. saccharalis. Além disso, é reconhecido que a produção de progênie depende do grau de competição entre irmãos em desenvolvimento dentro de um hospedeiro compartilhado (RIDDICK, 2008). A progênie pode variar conforme a espécie hospedeira, como ocorreu com $T$. diatraeae emergidos de pupas de $S$. frugiperda, A. gemmatalis, D. saccharalis, H. virescens,
T. arnobia, H. paulex e $S$. cosmioides (PARON; BERTI FILHO, 2000; PASTORI et al., 2012; ZACHÉ et al., 2012). Assim, para otimizar a emergênica da progênie, a fêmea parasitoide deverá variar quantidade de postura dos ovos em um hospedeiro, dependendo do tamanho ou da qualidade do mesmo (BENZEMER; MILLS, 2003). Há evidências que algumas espécies de parasitoides, depositam mais ovos em hospedeiros maiores (GODFRAY, 1994; HARVEY, 2000; ZAVIEZO; MILLS, 2000; WEST et al., 2001), fortalecendo os nossos achados em B. mori.

O ciclo de vida (ovo-adulto) e os tamanhos da cápsula cefálica e do corpo de machos e fêmeas de $T$. diatraeae foram semelhantes em ambos os hospedeiros testados, sugerindo que o hospedeiro alternativo é de qualidade e não irá interferir no período de desenvolvimento e no tamanho do parasitoide. O ciclo de vida apresentou duração semelhante quando o mesmo foi criado no hospedeiro natural. A duração do ciclo de vida de um parasitoide depende de vários fatores, dentre eles a espécie hospedeira (ZAGO et al., 2006).

A longevidade de machos e fêmeas foi diferente nos indivíduos emergidos nos dois hospedeiros. Esse resultado pode estar relacionado com a disponibilidade de alimento dos hospedeiros, pois pupas de B. mori são maiores e oferecem maior quantidade de energia, o que pode explicar a maior longevidade de fêmeas e 
machos em comparação aos adultos emergidos em $D$. saccharalis. Sabe-se que o hospedeiro não só afeta o desenvolvimento na fase imatura do parasitoide, como também pode afetar o seu comportamento e fisiologia na fase adulta (MEHRNEJAD, 2003).

A razão sexual de $T$. diatraeae em pupas de $D$. saccharalis e $B$. mori foi semelhante e elevada, o que demonstra a adequabilidade do hospedeiro alternativo para criação do parasitoide. Fêmeas parasitoides são as principais responsáveis pelo parasitismo e pela produção de descendentes (MATOS NETO et al., 2004), e a obtenção de um elevado número de fêmeas na progênie é importante para sistemas de criação massal, experimentos de laboratórios e seleção de indivíduos para liberação no campo (AMALIN et al., 2005).

Trichospilus diatraeae apresenta em laboratório desenvolvimento reprodutivo satisfatório (parasitismo, emergência, progênie por pupa, razão sexual, longevidade de fêmeas e machos) em pupas de $B$. mori o que demonstra a adequabilidade desse hospedeiro, bem como a capacidade do parasitoide de parasitar e adequarse a diferentes hospedeiros, pois com base nessas informações é possível estabelecer um sistema de criação desse agente de controle biológico em laboratório ou em biofábricas, para manutenção de criações ou quando ocorre a falta ou a queda na produção do hospedeiro natural poderá utilizar-se outro hospedeiro alternativo sem comprometer no desenvolvimento e qualidade do parasitoide.

\section{Agradecimentos}

Ao Conselho Nacional de Desenvolvimento Científico e Tecnológico (CNPq), à Coordenação de Aperfeiçoamento de Pessoal de Nível Superior (CAPES) e à Empresa de Fiação de Seda $\square$ BRATAC S/A.

\section{Referências}

ABBOTT, W. S. A method of computing the effectiveness of an insecticide. Journal of Economic Entomology, Riverside, v. 18, p. 265-267, 1925.

ANDRADE, G. S.; SERRÃO, J. E.; ZANUNCIO, J. C.; ZANUNCIO, T. V.; LEITE, G. L. D.; POLANCZYK, R. A. Immunity of an Alternative host can be overcome by higher densities of its parasitoids Palmistichus elaeisis and Trichospilus diatraeae. Plos One, Cambridge, v. 5, n. 10, p. 13231, 2010.

AMALIN, D. M.; PENA, J. E.; DUNCAN, R. E. Effects of host age, female parasitoid age, and host plant on parasitism of Ceratogramma etiennei (Hymenoptera: Trichogrammatidae). Florida Entomologist, Lutz, v. 88, n. 1, p. 77-82, 2005.

BEZEMER, T. M.; MILLS, N. J. Clutch size decisions of a gregarious parasitoid under laboratory and field conditions. Animal Behaviour, St Andrews, v. 66, p. 1119-1128, 2003.

DIAS, N. da S.; PARRA, J. R. P.; LIMA, T. C. da C. Seleção de hospedeiro alternativo para três espécies de tricogramatídeos neotropicais. Pesquisa Agropecuária Brasileira, Brasília, v. 11, n. 43, p. 1467-1473, 2008.

GODFRAY, H. C. J. Parasitoids, Behavioral and Evolutionary Ecology. Princeton: Princeton University Press, 1994. 473 p.

HARVEY, J. A. Dynamic effects of parasitism by an endoparasitoid wasp on the development of two host species: implications for host quality and parasitoid fitness. Ecological Entomology, London, v. 25, n. 3, p. 267-278, 2000.

IHAKA, R.; GENTLEMAN, R. A language for data analysis and graphics. Journal of Computational and Graphical Statistics, Alexandria, v. 5, p. 299-314, 1996.

MATOS NETO, F. C.; CRUZ, I.; ZANUNCIO, J. C.; SILVA, C. H. O.; PICANÇO, M. C. Parasitism by Campoletis flavicincta on Spodoptera frugiperda in corn. Pesquisa Agropecuária Brasileira, Brasília, v. 39, n. 11, p. 1077-1081, 2004.

MEHRNEJAD, M. R. The influence of host species on some biological and behavioural aspects of Dibrachys boarmiae (Hymenoptera: Pteromalidae), a parasitoid of Kermania pistaciella (Lepidoptera: Tineidae). Biocontrol Science and Technology, Abingdon, v. 13, p. 219-229, 2003.

MELO, R. L.; PRATISSOLI, D.; POLANCZYK, R. A.; TAVARES, M.; MILANEZ, A. M.; MELO, D. F. Ocorrência de Trichospilus diatraeae (Hymenoptera.: Eulophidae) em broca-das-cucurbitáceas, no Brasil. Horticultura Brasileira, Campinas, v. 29, n. 2, p. 228230, 2011.

OLIVEIRA, M. A. S.; JUNQUEIRA, N. T. V.; ICUMA, I. M.; ALVES, R. T.; OLIVEIRA, J. N. S.; ANDRADE, G. A. Incidência de danos da broca do fruto da graviola no Distrito Federal. Planaltina: Embrapa Cerrados, n. 51, 2001. (Comunicado TécnicoEmbrapa).

PANIZZI, A. R.; PARRA, J. R. P. Ecologia nutricional de insetos e suas implicações no manejo de pragas. Brasília: Editora Manole/ CNPq, 1991. 359 p.

PARON, M. R.; BERTI-FILHO, E. Capacidade reprodutiva de Trichospilus diatraeae (Hymenoptera: Eulophidae) em pupas de diferentes hospedeiros (Lepidoptera). Scientia Agricola, Piracicaba, v. 57, n. 2, p. 355-358, 2000.

PARRA, J. R. P. Técnicas de criação de insetos para programa de controle biológico. 6. ed. Piracicaba: ESALQ/FEALQ, 2007. $134 \mathrm{p}$.

PARRA, J. R. P.; BOTELHO, P. S. M.; CORRÊA-FERREIRA, B. S.; BENTO, J. M. S. Controle biológico no Brasil: parasitoides e predadores. São Paulo: Manole, 2002. 635 p.

PASTORI, P.L.; PEREIRA, F. F.; ANDRADE, G. S.; SILVA, R. O.; ZANUNCIO, J. C.; PEREIRA, A. I. A. Reproduction of 
Trichospilus diatraeae (Hymenoptera: Eulophidae) in pupae of two lepidopterans defoliators of eucalypt. Revista Colombiana de Entomología, Bogotá, v. 38, n. 1, p. 91-93, 2012.

PEREIRA, F. F.; ZANUNCIO, J. C.; PASTORI, P. L.; PEDROSA, A. R. P.; OLIVEIRA, H. N. de. Parasitismo de Palmistichus elaeisis (Hymenoptera: Eulophidae) em hospedeiro alternativo sobre plantas de eucalipto em semi-campo. Revista Ciência Agronômica, Fortaleza, v. 4, n. 41, p. 715-720, 2010 a.

PEREIRA, F. F.; ZANUNCIO, J. C.; SERRÃO, J. E.; PASTORI, P. L.; RAMALHO, F. S. Reproductive performance of Palmistichus elaeisis Delvare e La Salle (Hymenoptera: Eulophidae) with previously refrigerated pupae of Bombyx mori L. (Lepidoptera: Bombycidae). Brazilian Journal of Biology, São Carlos, v. 69, p. 631-637, 2009.

PEREIRA, F. F.; ZANUNCIO, J. C.; SERRÃO, J. E.; ZANUNCIO, T. V.; PRATISSOLI, D.; PASTORI, P. L. The density of females of Palmistichus elaeisis Delvare and LaSalle (Hymenoptera: Eulophidae) affects their reproductive performance on pupae of Bombyx mori L. (Lepidoptera: Bombycidae). Anais da Academia Brasileira de Ciências, São Paulo, v. 2, n. 82, p. 323-331, 2010b.

PEREIRA, F. F.; ZANUNCIO, J. C.; TAVARES, M. T.; PASTORI, P. L.; JACQUES, G. C. New record of Trichospilus diatraeae (Hymenoptera: Eulophidae) as a parasitoid of the eucalypt defoliator Thyrinteina arnobia (Lepidoptera: Geometridae) in Brazil. Phytoparasitica, Rehovot, v. 36, n. 3, p. 304-306, 2008.

PRATISSOLI, D.; THULER, R. T.; ANDRADE, G. S.; ZANOTTI, L. C. M.; SILVA, A. F. Estimativa de Trichogramma pretiosum para o controle de Tuta absoluta em tomateiro estaqueado. Pesquisa Agropecuária Brasileira, Brasília, v. 40, p. 715-718, 2005.

RIDDICK, E. W. Sting frequency and progeny production of labcultured Cotesia marginiventris. Biological Control, Cambridge, v. 53, p. 295-302, 2008.

WANG-DUN, B.; ZAHANG, C. A review on the nutritive value of silk worm pupae and its exploitation. Entomological Knowledge, Beijing, v. 41, p. 418-421, 2004.

WEST, S. A.; FLANAGAN, K. E.; GODFRAY, H. C. J. Variable host quality, life-history invariants, and the reproductive strategy of a parasitoid wasp that produces single sex clutches. Behavioral Ecology, Göttingen, v. 12, p. 577-583, 2001.
ZACHÉ, B.; WILCKEN, C. F.; DACOSTA, R. R.; SOLIMAN, E. P. Trichospilus diatraeae Cherian \& Margabandhu, 1942 (Hymenoptera: Eulophidae), a new parasitoid of Melanolophia consimilaria (Lepidoptera: Geometridae). Phytoparasitica, Rehovot, v. 38, n. 3, p. 355-357, 2010a.

ZACHÉ, B.; WILCKEN, C. F.; ZACHÉ, R. R. C.; SOLIMAN, E. P.; SAN ROMAN, M. L. L. Trichospilus diatraeae Cherian \& Margabandhu, 1942 (Hymenoptera: Eulophidae), un nuevo parasitoide de Hypsipyla grandella (Zeller, 1848) (Lepidoptera: Pyralidae). Idesia, Arica, v. 28, p. 111-114, 2010 b.

ZACHÉ, B.; WILCKEN, C. F.; ZACHÉ, R. R. C.; SOUZA, N. M. Novo registro de Trichospilus diatraeae Cherian \& Margabandhu, 1942 (Hymenoptera: Eulophidae), como parasitoide de Spodoptera cosmioides Walker, 1858 (Lepidoptera: Noctuidae) no Brasil. Biota Neotropica, Campinasm, v. 12, n. 1, p. 319-322, 2012.

ZACHÉ, B.; ZACHÉ, R. R. C.; SOLIMAN, E. P.; WILCKEN, C. F. Evaluation of Trichospilus diatraeae (Hymenoptera: Eulophidae) as parasitoid of the eucalyptus defoliator Euselasia eucerus (Lepidoptera: Riodinidae). International Journal Tropical Insect Science, Cambridge, v. 31, n. 1-2, p. 118-121, 2011a.

ZACHÉ, B.; ZACHÉ, R. R. C.; SOUZA, N. M.; DIAS, T. K. R.; WILCKEN, C. F. New record of Trichospilus diatraeae Cherian \& Margabandhu, 1942 (Hymenoptera: Eulophidae) parasitizing Sarsina violascens (Herrich-Schaeffer, 1856) (Lepidoptera: Lymantriidae) in Brazil. Journal Plant Protection Resistence Research, Berlin, v. 51, p. 420-422, 2011b.

ZAGO, H. B.; PRATISSOLI, D.; BARROS, R.; GONDIM JR., M. G. C. Biologia e exigências térmicas de Trichogramma pratissolii Querino \& Zucchi (Hymenoptera: Trichogrammatidae) em hospedeiro alternativos. Neotropical Entomology, Piracicaba, v. 35, p. 377-381, 2006.

ZANUNCIO, J. C.; PEREIRA, F. F.; JACQUES, G. C.; TAVARES, M. T.; SERRÃO, J. E. Tenebrio molitor Linnaeus (Coleoptera: Tenebrionidae), a new alternative host to rear the pupae parasitoid Palmistichus elaeisis Delvare \& LaSalle (Hymenoptera: Eulophidae). The Coleopterisits Bulletin, Washington, v. 62, n. 1, p. 64-66, 2008.

ZAVIEZO, T.; MILLS, N. J. Factors influencing the evolution of clutch size in a gregarious insect parasitoid. Journal of Animal Ecology, London, v. 69, p.1047-1057, 2000. 